\title{
Daily use of a muscle pump activator device reduces duration of hospitalization and improves early graft outcomes post-kidney transplantation: A randomized controlled trial
}

\author{
Wen Xie, MD ${ }^{1}$; Max A. Levine, $\mathrm{MD}^{1,2}$; Shahid Aquil, MD ${ }^{1}$; Katharine Pacoli, $\mathrm{BSc}^{3}$; \\ Rafid Al-Ogaili, MD ${ }^{1}$; Patrick P. Luke MD ${ }^{1,2,3}$; Alp Sener MD, PhD ${ }^{1,2,3,4}$ \\ ${ }^{1}$ Departments of Surgery, Schulich School of Medicine \& Dentistry, St. Joseph's Hospital, London, ON, Canada; \\ ${ }^{2}$ Multi-Organ Transplant Program London Health Sciences Centre, London Health Sciences Centre, London, ON, \\ Canada; ${ }^{3}$ Matthew Mailing Centre for Translational Transplant Studies, London Health Sciences Centre, London, \\ ON, Canada; ${ }^{4}$ Department of Microbiology \& Immunology, Schulich School of Medicine \& Dentistry \\ Western University, London, ON, Canada
}

Funding: This study is support by an unrestricted investigator-initiated grant from Firstkind Ltd

Cite as: Can Urol Assoc J 2020 July 27; Epub ahead of print. http://dx.doi.org/10.5489/cuaj.6487

Published online July 27, 2020

$* * *$

\begin{abstract}
Introduction: Kidney and simultaneous pancreas-kidney (SPK) transplant recipients can have prolonged postoperative hospitalization due to edema. Thrombo-embolic-deterrent (TED) stockings with intermittent pneumatic compression devices (TED+IPC) have been used to improve venous return during the perioperative period. The objective of this trial was to evaluate the effects of TED+IPC vs. muscle pump activator (MPA) devices on factors that could reduce postoperative complications and duration of hospitalization.

Methods: In this single-center, prospective, randomized, controlled trial, 221 kidney and SPK transplant recipients were randomized to either wearing TED+IPC or MPA for six days postoperatively. Groups were compared with respect to postoperative urine output, lower limb edema, weight, days in hospital, mobility, serum creatinine, delayed graft function, need for dialysis, and lower extremity blood flow.

Results: Patients in the MPA group had significantly higher urine output and less increase in mid-calf leg circumference and weight gain compared to the TED+IPC group $(p=0.003$, $\mathrm{p}=0.001$, and $\mathrm{p}=0.003$, respectively). The MPA group also experienced shorter hospitalization $(p=0.038)$, higher femoral vein velocity $(p=0.001)$, and took more steps $(p=0.009)$. Incidence of delayed graft function $(p=0.72)$ and number of dialysis runs $(p=0.39)$ was not different between study groups. Subgroup analysis of primary endpoints in donation after cardiac death recipients and SPK recipients did not yield any significance between the study arms.
\end{abstract}


Conclusions: Postoperative use of the MPA device increases urine output, decreases leg edema, minimizes weight gain, and decreases duration of hospitalization after kidney transplantation. A larger and longer-term trial is needed to evaluate the impact on graft function.

\section{Introduction}

Kidney and simultaneous pancreas-kidney (SPK) transplantations can significantly reduce mortality and improve quality of life in patients with end stage renal disease (ESRD) and select patients with diabetes and ESRD, respectively ${ }^{1-5}$. Post-operatively, recipients may require a prolonged period of hospitalization secondary to a number of factors, including delayed-graft function (DGF), delayed mobilization, and edema ${ }^{6-8}$. Edema may also contribute to other postoperative complications including impaired wound healing and ileus $s^{9,10}$.

Fluid management after renal transplantation can be challenging. The need to provide adequate pre-load to perfuse the newly transplanted allograft can conflict with efforts to avoid volume overload. The latter condition can be slow to correct, especially if the allograft is experiencing slow graft function or DGF. Management of lower-limb circulation and postoperative lower-limb edema is of special interest in kidney and SPK transplantation given the site of implantation for the allograft vessels. The benefits of improving post-operative lowerlimb circulation and correcting lower-limb edema has been demonstrated in trauma orthopedic surgery ${ }^{11}$ and lower-limb revascularization surgery ${ }^{12}$, where improvements in mobility and surgical outcome have been demonstrated.

TED stockings and intermittent pneumatic compression devices (TED+IPC) are the established standard of care for management of post-operative lower-limb edema and venous stasis $^{13}$. The devices can be uncomfortable to wear, and the large external pneumatic pump inhibits patient mobility. Muscle pump activator (MPA) devices have been found to be an effective alternative to IPC devices for management of post-operative lower-limb edema ${ }^{14}$. The geko $^{\text {TM }}$ (Firstkind Ltd, UK) device is a portable, wireless MPA device, worn as a band over the legs bilaterally, just inferior to the fibular heads (Fig. 1). The resulting simultaneous contraction of tibialis, peroneus longus and lateral gastrocnemius muscles compress the deep veins to increase blood flow in these vessels. In addition to greater improvements in both macrocirculation ${ }^{15}$ and microcirculation ${ }^{14}$ of the lower-limb in comparison to standard IPC pumps, the device is smaller, portable and more comfortable to wear ${ }^{16}$. Despite various studies demonstrating equal or greater efficacy in hemodynamic parameters, there is very limited highquality evidence for the effects of MPA on clinically relevant measures ${ }^{17}$. This randomizedcontrolled trial aims to elucidate the impact of MPA on lower-limb edema, renal function, and length of hospitalization in relation to standard TED+IPC after kidney and SPK transplantation. We hypothesized that the geko ${ }^{\mathrm{TM}}$ device used in the post-operative setting following kidney and 
SPK transplants would lead to improved lower limb edema resulting in a decreased length of stay in hospital.

\section{Methods}

\section{Study design}

This study was designed as an open-labelled, single-centered, prospective, randomizedcontrolled trial. The study was conducted in accordance to the CONSORT 2010 Guidelines and followed the principles of the Declaration of Helsinki. Institutional review approval was provided by the Western University Research Ethics Board (Protocol number 103618, ClinTrials.gov NCT01860820).

\section{Participants}

Patients undergoing kidney and SPK transplantation at London Health Sciences Center were eligible for the study if they were $\geq 18$ years old. Exclusion criteria include: age $<18$ years old, history of DVT, history of leg amputation, BMI $>36$, use of an implantable cardiac defibrillator, presence of deep brain stimulators, other contraindications to use of electrical stimulation devices, lack of ability to understand the risks and benefits of the study, and those who cannot tolerate the MPA device stimulation.

Induction immunosuppressive therapy consisted of antithymocyte globulin $(5-8 \mathrm{mg} / \mathrm{kg}$ IV) or Basiliximab (20mg IV on post-operative day (POD) 0 and 4) depending on the recipient's immunological risk. Maintenance immunosuppressive regimen consisted of prednisone, tacrolimus and mycophenolic acid, and were initiated while in hospital for all recipients. Serum drug levels were monitored closely, and dosages were adjusted according to accepted practices. Recipients of a renal transplant have their urinary catheters removed on POD 5 as a program standard. In cases when patients were discharged home prior to POD 5, their catheters were removed on the day of discharge.

\section{Randomization and interventions}

Patients deemed eligible for the study provided informed consent and underwent randomization prior to their transplant surgery. Randomization was performed by a study coordinator, independent of the patient's clinical care, using an online computer generator sequence. Participants were placed into either the control (TED+IPC) or interventions (MPA, geko ${ }^{\mathrm{TM}}$, Firstkind Ltd, UK) arm based on randomization. The surgical team was blinded to patient's group allocation at the time of surgery.

As per institutional standard, TED+IPC was used for intraoperative deep vein thrombosis (DVT) prophylaxis for both groups. All participants were also placed on dalteparin 5000IU subcutaneous injection daily for DVT prophylaxis starting on POD 1. Patients assigned to the MPA group were then switched over the geko ${ }^{\mathrm{TM}}$ device on post-operative day 1 . The device was properly fitted and adjusted for stimulation of the common peroneal nerve according to manufacturer's instructions for use. Patients randomized to the control arm continued to wear the 
TED+IPC device post-operatively. On post-operative day 6 , or at the time of discharge if duration of hospitalization was less than 6 days, both groups had their devices removed. Postoperative care and discharge planning was performed by an independent nephrology team blinded to the randomization and uninvolved with the trial.

\section{Outcomes}

Co-primary endpoints were lower-limb edema and total urine output between POD 1-6. Lower limb edema is reflected by the difference in calf circumference between POD 1 and 6, or day of discharge, which ever came first. Calf circumference is measured using a tape measure at $15 \mathrm{~cm}$ below the patella's midpoint. Serum creatinine was used to directly report renal function. Based on a pilot study performed at our center suggesting a difference in lower limb edema between the study groups, we sought to study a more clinically relevant metric as part of our primary outcome measure. We hypothesized that reduction in lower-limb circumference would translate into increased urine output, which is relevant to the early post-transplant period. Difference in patient weight was measured in kilograms between POD 1 and 6, or day of discharge, which ever came first.

Secondary endpoints included length of stay, occurrence of DGF defined as the need for dialysis within one week of transplant, number of dialysis sessions post operatively, renal blood flow, and mobility. Incidence of DGF was assessed dichotomously and further delineated by number of dialysis runs needed during the post-operative hospitalization period. Peak femoral vein velocity was measured in centimeter per second on doppler ultrasound of the renal allograft on POD 6. During the post-operative period, participants in both groups wore pedometers and monitored their physical activity between POD 2 to 5. Post-operative incidence of DVT was also monitored.

\section{Statistical analysis}

Based on pilot study data, we expected the mean calf circumference in the TED+SCD group to increase by $3.5 \mathrm{~cm}$ with a SD of 1.5 . Given there is no defined minimal clinically important difference for calf circumference, we used the statistical convention of $1 / 2 \mathrm{SD}$ to estimate this value. Therefore, we determined that in the MPA group an increase in calf-circumference to 2 would provide a moderate effect size of 0.5 . With a desired power of $90 \%$, we determined that sample size required for each group would be 86 . We repeated this power calculation rationale for the co-primary end-point of urine output from POD 1 to 6 using a baseline urine output in the TED+SCD group of $12.5 \mathrm{~L}$ with a SD of $8.4 \mathrm{~L}$ based on pilot study data. We arrived at the same required sample size of 86 . We then accounted for $\sim 20 \%$ attrition arriving at 103 patients per group. Statistical tests were done with per protocol analysis. GraphPad Prism was used to conduct a statistic analysis using a Students' t-test for independent data groups where $\mathrm{p}<0.05$ representing the point of statistical significance. The normalcy of distribution of demographics and patient characteristics with outlier detection were assessed by Shapiro-Wilk's test. Data are reported as mean \pm standard deviation (SD) unless otherwise specified. 


\section{Results}

Between September 2015 to September 2017, 259 kidney and SPK transplantation were completed at London Health Sciences Center. A total of 221 recipients were determined to be eligible for the study, provided informed consent, and were subsequently randomized (Kidney transplant=204, SPK transplant=17; Donation after Brain Death=109, Living Donor=52, Donation after Cardiac Death=60). After randomization, two patients originally assigned to the intervention arm could not tolerate the stimulation from the MPA device and one participant in the intervention arm did not experience any muscle contraction despite optimization of device placement. These participants subsequently crossed over to the control arm within the first day of using the device and continued in the study until completion and were analyzed according to treatment received. One-hundred and eleven were eventually analyzed in the control arm and 110 to the intervention arm in the per protocol analysis.. All participants adhered to wearing their device for the whole study period and there were no patients lost to follow up as relevant data collection was performed during hospitalization. Baseline characteristics and co-morbidities were similar between groups (Table $1 \&$ Table 2).

\section{Primary endpoints}

All patients experienced an increase in lower-limb circumference with post-operative fluid administration between the identified time points. The MPA group had an increase in calf circumference of $2.5 \pm 1.5 \mathrm{~cm}$. This was significantly lower than the TED+IPC group, which had a mean increase in calf circumference of $3.6 \pm 1.5 \mathrm{~cm}(\mathrm{p}=0.001$, Fig. 2). Similarly, the change in patient weight between POD 1 and 6 was also significantly less in the MPA group compared to the TED+IPC group (4.06 $\pm 2.3 \mathrm{~kg}$ vs $5.18 \pm 2.8 \mathrm{~kg}, \mathrm{p}=0.003$, Fig. 3 ).

Patients who wore the MPA device made on average $15.99 \pm 8.8 \mathrm{~L}$ of urine between POD 1-6 in contrast to an average of $12.60 \pm 8.4 \mathrm{~L}$ made by TED + IPC group ( $p=0.003$, Table 3 ). Further analysis categorizing recipients by donor type also found a significant difference between the MPA and TED+IPC group in living and DBD donor kidneys $(23.99 \pm 5.42 \mathrm{~L}$ vs $18.90 \pm 7.64 \mathrm{~L}, \mathrm{p}=0.009 ; 19.52 \pm 5.36 \mathrm{~L}$ vs $12.85 \pm 7.13 \mathrm{~L}, \mathrm{p}<0.001$, Table 4$)$. However, serum creatinine on POD 6 was not significantly different between the two groups $(162 \pm 171 \mu \mathrm{mol} / \mathrm{L}$ vs $170 \pm 146 \mu \mathrm{mol} / \mathrm{L}, \mathrm{p}=0.45)$. Although recipients who received DCD donor kidneys and used the MPA device made more urine than their TED+IPC counterparts, there was no statistical difference seen $(5.60 \pm 4.01 \mathrm{~L}$ vs $4.31 \pm 4.33 \mathrm{~L}, \mathrm{p}=0.27)$.

In the SPK subpopulation, the differences in our co-primary endpoints between groups were not observed.

\section{Secondary endpoints}

The duration of hospitalization post transplantation varied between 4-30 days with the average length of stay being 8.3 days after kidney only transplant and 14.6 days after SPK transplant 
(Fig. 4). Overall, the MPA group had a significantly shorter hospital stay compared to the TED+IPC group ( $8.15 \pm 3.5$ days vs. $9.36 \pm 5.0$ days, $\mathrm{p}=0.038$ ).

With the MPA or TED+IPC devices in place, Doppler ultrasound on POD 6 showed higher femoral vein velocity in the MPA group $(18.90 \pm 4.4 \mathrm{~cm} / \mathrm{s})$ compared to the TED+IPC group $(14.41 \pm 5.1 \mathrm{~cm} / \mathrm{s}, \mathrm{p}=0.001)$. Despite improved venous blood flow, use of the MPA device was not associated with decreased rates of DGF $(24 \%$ vs $22 \%, \mathrm{p}=0.72)$ or number of dialysis runs ( $56 \mathrm{vs} 73, \mathrm{p}=0.39$ ). A total of 80 participants wore pedometers to monitor their level of mobility on POD 2-5 (IPC = 45, MPA = 35). Those who wore the MPA device walked an average of $1231 \pm 189$ steps, indicating a greater level of mobility compared to the control arm ( $1099 \pm 249$ steps, $p=0.009$, Fig. 5). There were 2 recipients in the MPA group and 1 recipient in the IPC group who developed lower limb DVTs. No statistical analysis was performed as the study was not powered to assess differences in DVT incidence rates.

\section{Discussion}

With increasing health care costs and significant portions of the overall cost accruing from inpatient care, many surgical specialities have made efforts towards shortening the duration of post-operative hospitalization ${ }^{18,19}$. Since being established in the early 2000s, Enhanced Recovery after Surgery (ERAS) has permeated through many surgical fields as an evidencebased, multimodal, multidisciplinary approach for care of the surgical patient ${ }^{20,21}$. One of the tenants of ERAS is minimizing fluid shifts ${ }^{22}$ such that the sequelae of fluid overload can be avoided $^{23,24}$. This can be particularly difficult in transplant recipients given the pre-existing electrolyte imbalance ${ }^{25}$ and generous fluid administration sometimes required to keep adequate intravascular volume to perfuse the allograft. Evidence has shown that external compression of the lower limbs to be effective in preventing the redistribution of fluids from the intravascular to extravascular compartments, thereby decreasing fluid demands and maintaining hemodynamic stability $^{26}$.

In our study, we compared two devices that, through different mechanisms, compress the venous system of the lower limbs. ${ }^{13,27}$ Similar to previous findings, ${ }^{16,28}$ participants who used the MPA device were found to have significantly less lower limb edema in the perioperative period. This has been explained by previous studies showing that stimulation of the intrinsic muscle pumps is more effective than external compression for increasing venous return ${ }^{14}$ and preventing extravascular redistribution of fluids around time of surgery ${ }^{13}$. It stands to reason that with improved venous return from the lower limbs, there is better perfusion to the newly transplanted kidney to facilitate more urine production and as a result, less fluid retention. This is reflected in our findings of higher urine output and less weight gain in participants who used the MPA device.

Subgroup analysis of recipients in the MPA group who received DCD donor kidneys, on average, made $1 \mathrm{~L}$ more than their TED+IPC counterparts. This was not statistically significant, although the sample size was small and the incidence of DGF in the DCD cohort was high (data not shown). As a result, urine output in the first week post-transplantation, in both groups, was 
low. In the early post-operative course, urine output has been found to be an independent predictive factor on graft survival and should therefore be optimized ${ }^{29}$. Our results do suggest that the MPA device may have some clinical impact on fluid shifts as the calf size measurements was trending towards statistical significance in the DCD cohort. Whether these non-significant improvements in fluid mobilization from the use of the MPA device impacts long term graft function is unknown but warrants further investigations with longer follow-up.

We were able to demonstrate that the use of an MPA device was associated with a shorter length of hospitalization compared to the TED+IPC devices after kidney transplantation. Many factors are considered when determining a patient's readiness to be discharged, including clinical parameters such as ambulation, tolerance of oral intake, bowel motility and pain control. In the subset of participants who wore pedometers, individuals assigned to use the MPA device was also found to have improved post-operative mobility. This could be related to the significant reduction in lower extremity edema which may increase the ease and comfort of ambulation. The portable nature of the MPA device also promotes ambulation as it allows for greater freedom and independence to mobilize without having to manage the pneumatic pump device used with $\mathrm{IPCs}^{30}$. Although evidence on effect of ambulation on gut mobilization is scarce ${ }^{31-33}$, early mobilization is known to prevent perioperative pulmonary and thromboembolic complications $^{34,35}$ and thus prevent additional days of hospitalization. We have also shown in a pilot study that significantly fewer patients described "some level of discomfort" on a patient satisfaction survey compared to TED+IPC $(13 \% \text { vs } 57 \%, p=0.003)^{36}$. This knowledge further supports the use of the MPA device.

Finally, we did not find any significance in our study parameters during secondary analysis of our SPK transplant cohort. This may be due to the small sample size of this subgroup. The lack of benefit may also be attributable to the more extended and complex nature of the surgery. The duration of the operation is longer for SPK recipients given the additional vascular and bowel anastomosis. This can cause a greater stress response with release of more cytokines leading to fluid redistribution, similar to what is seen in other major abdominal surgeries ${ }^{37}$. SPK recipients would generally be without oral fluid intake post-operatively for longer, with potential for increased fluid losses through a nasogastric tube. This leads to more variable fluid prescription, making it more difficult to assess the effect of the MPA device on urine output, lower limb edema and patient weight.

To our knowledge, this is the first randomized-controlled trial looking at the effect of an MPA device on decreasing length of post-operative hospitalization. A significant strength of our study was accrual of an adequate sample size determined a priori, without attrition. Clinically relevant and objective measures were assessed, giving our results external validity.

This study is limited by its single center design with short-term follow-up of the participants. The open-label design also created some limitations as the patient and care teams were not blinded to the modality of lower limb device due to the vastly discrepant size and appearance of the two devices. This represents a potential source of bias for post-operative 
decisions with regards to ambulation and discharge. Patients are typically mobilized with nursing staff help on POD 2 as per institutional care pathway. Physiotherapy is enlisted when patients have difficulties ambulating due to pre-operative mobility limitations, or for newly identified mobility concerns. Since the care pathway implores mobilization on day 2, and TED+IPC represents the standard of care, the care teams are not expected to significantly alter the enthusiasm to ambulate patients with TED+IPC relative to those with the MPA devices. Another limitation of the study design is a lack of documentation of perioperative fluid administration. However, fluid administration in the early postoperative period was protocolized so all patients were exposed to the same decisions for fluid prescription. The overall change in patient weight is a direct reflection of total fluid given in relation to diuresis. Thus, even though fluid administration to the MPA group may have been higher, there was less weight gain overall, and less low limb edema which would be consistent with less fluid shift from the intravascular to extravascular components. Finally, although the incidence of DVT was low overall, there were 2 incidences of DVT in the MPA group compared to 1 incidence in the TED+IPC group. Without adequate power to study this outcome, it is possible that the MPA device is less effective at preventing DVT formation compared to TED+IPC although previously published reports have not found evidence to support this ${ }^{17}$.

\section{Conclusions}

Post-operative use of an MPA device decreases duration of hospitalization after kidney transplantation compared to when TED+IPC is used. This may be attributable to improved maintenance of intravascular volume leading to improved renal blood flow to the transplant allograft and thus increased urine output and decreased fluid retention. Further studies looking at long term outcomes and with focus on the DCD kidney transplant population are needed. 


\section{References}

1. Ojo AO, Meier-Kriesche H-U, Hanson JA, et al. The Impact of Simultaneous PancreasKidney Transplantation on Long-Term Patient Survival. Transplantation. 2001;71(1):8289. doi:10.1097/00007890-200101150-00014

2. Tyden G, Tollemar J, Bolinder J. Combined Pancreas and Kidney Transplantation Improves Survival in Patients with End-Stage Diabetic Nephropathy. Clin Transplant. 2000;14(5):505-508. doi:10.1034/j.1399-0012.2000.140510.x

3. Rabbat CG, Thorpe KE, Russell JD, Churchill DN. Comparison of Mortality Risk for Dialysis Patients and Cadaveric First Renal Transplant Recipients in Ontario, Canada. $J$ Am Soc Nephrol. 2000;11(5):917-922. http://www.ncbi.nlm.nih.gov/pubmed/10770970.

4. Wolfe RA, Ashby VB, Milford EL, et al. Comparison of Mortality in All Patients on Dialysis, Patients on Dialysis Awaiting Transplantation, and Recipients of a First Cadaveric Transplant. N Engl J Med. 1999;341(23):1725-1730. doi:10.1056/NEJM199912023412303

5. Oniscu GC, Brown H, Forsythe JLR. Impact of Cadaveric Renal Transplantation on Survival in Patients Listed for Transplantation. J Am Soc Nephrol. 2005;16(6):18591865. doi:10.1681/asn.2004121092

6. Ounissi M, Cherif M, Abdallah T Ben, et al. Risk Factors and Consequences of Delayed Graft Function. Saudi J Kidney Dis Transpl. 2013;24(2):243-246.

7. Ho CK., Sun M., Au TW., Chiu CSW. Pneumatic Pump Reduces Leg Wound Complications in Cardiac Patients. Asian Cardiovasc Thorac Ann. 2006;14(6):452-457. http://www.embase.com/search/results?subaction=viewrecord\&from=export\&id=L44883 672\%5Cnhttp://sfx.aub.aau.dk/sfxaub?sid=EMBASE\&issn=02184923\&id=doi:\&atitle=P neumatic + pump + reduces + leg + wound + complications + in + cardiac + patients $\&$ stitle $=$ Asian + Cardiovasc. + Thorac. + .

8. Van Beijsterveld CA, Bongers BC, Den Dulk M, Van Kuijk SMJ, Dejong KCH, Van Meeteren NLU. The association between preoperative physical functioning and shortterm postoperative outcomes: a cohort study of patients undergoing elective hepatic resection. HPB. 2019. doi:https://doi.org/10.1016/j.hpb.2019.02.009

9. Boeckxstaens GE, De Jonge WJ. Neuroimmune mechanisms in postoperative ileus. Gut. 2009;58(9):1300-1311. doi:10.1136/gut.2008.169250

10. Mitchell G, Hucker T, Venn R, et al. Pathophysiology and clinical implications of perioperative fluid excess (multiple letters) [1]. Br J Anaesth. 2003;90(3):395-396. doi:10.1093/bja/aeg526

11. Winge R, Bayer L, Gottlieb H, Ryge C. Compression Therapy After Ankle Fracture Surgery : A Systematic Review. Eur J Trauma Emerg Surg. 2017;43(4):451-459. doi:10.1007/s00068-017-0801-y

12. Slaa A, Dolmans D, Ho G. Treatment Strategies and Clinical Aspects of Lower Limb Edema Following Peripheral Bypass Surgery. Phlebology. 2014;29(1S):18-25. doi:10.1177/0268355514527689

13. Tessari M, Tisato V, Rimondi E, Zamboni P. Effects of Intermittent Pneumatic Compression Treatment on Clinical Outcomes and Biochemical Markers in Patients at Low Mobility with Lower Limb Edema. J Vasc Surg. 6(4):500-510. doi:10.1016/j.jvsv.2018.01.019 
14. Bahadori S, Immins T, Wainwright TW. The Effect of Calf Neuromuscular Electrical Stimulation and Intermittent Pneumatic Compression on Thigh Microcirculation. Microvasc Res. 2017;111:37-41. doi:10.1016/j.mvr.2017.01.001

15. Williams KJ, Moore HM, Davies AH. Haemodynamic Changes with the Use of Neuromuscular Electrical Stimulation Compared to Intermittent Pneumatic Compression. Phlebology. 2015;30(5):365-372. doi:10.1177/0268355514531255

16. Aquil S, Alharbi B, Navaratnam R, Sharma H, Luke PP, Sener A. Use of a Muscle Pump Activator Leads to Improved Lower Limb Edema, Lower Limb Blood Flow, and Urine Output Compared With Standard TED Stockings and Compression Devices Following Kidney Transplant: A Randomized Controlled Trial. Transplant Proc. 2019;7. doi:10.1016/j.transproceed.2019.04.032

17. Hajibandeh S, Hajibandeh S, Ga A, Scurr J, Torella F. Neuromuscular electrical stimulation for the prevention of venous thromboembolism ( Review ). Cochrane Database Syst Rev. 2017;(11). doi:10.1002/14651858.CD011764.pub2.www.cochranelibrary.com

18. OECD. Health spending (indicator). 2018. doi:10.1787/8643de7e-en

19. Fund TC. Inpatient Hospital Spending per capita. https://www.commonwealthfund.org/chart/2010/inpatient-hospital-spending-capita-2006. Published 2010.

20. Ljungqvist, Olle; Scott, Michael; Fearon K. 'Enhanced Recovery After Surgery A Review. Clin Rev Educ. 2017;152(3):292-298. doi:10.1001/jamasurg.2016.4952

21. T K, Niekowa B, Krasnicka M, Sadowski J. Enhanced Recovery After Kidney Transplantation Surgery. Transplant Proceedings,. 2016;48:1461-1465. doi:10.1016/j.transproceed.2015.11.037

22. Gustafsson UO, Scott MJ, Hubner M, et al. Guidelines for Perioperative Care in Elective Colorectal Surgery: Enhanced Recovery After Surgery (ERAS $\left.{ }^{\circledR}\right)$ ) Society Recommendations: 2018. World J Surg. 2019;43(3):659-695. doi:10.1007/s00268-0184844-y

23. Lobo DN, Bostock KA, Neal KR, Perkins AC, Rowlands BJ, Allison SP. Effect of Salt and Water Balance on Recovery of Gastrointestinal Function After Elective Colonic Resection : A Randomised Controlled Trial. Lancet. 2002;359:1812-1818.

24. Bragg D, El-sharkawy AM, Psaltis E, Maxwell-armstrong CA, Lobo DN. Postoperative Ileus : Recent Developments in Pathophysiology and Management. Clin Nutr. 2015;34(3):367-376. doi:10.1016/j.clnu.2015.01.016

25. Jerry Yee, MD; Raviprasenna Parasuraman; and Robert G. Narins M. Selective Review of Key Perioperative Renal-Electrolyte Disturbances in Chronic Renal Failure Patients. Chest. 1999;115(5 SUPPL.):125S-129S. doi:10.1378/chest.115.suppl

26. Kiefer N, Theis J, Putensen-himmer G, Hoeft A, Ph D, Zenker S. Peristaltic Pneumatic Compression of the Legs Reduces Fluid Demand and Improves Hemodynamic Stability. Anesthesiology. 2018;114(3):536-544.

27. Tucker AT, Maass A, Bain DS, et al. Augmentation of venous, arterial and microvascular blood supply in the leg by isometric neuromuscular stimulation via the peroneal nerve. Int J Angiol. 2010;19(1):31-37. doi:10.1055/s-0031-1278361

28. Ravikumar R, Williams KJ, Babber A, Lane TRA, Moore HM, Davies AH. Randomised Controlled Trial: Potential Benefit of a Footplate Neuromuscular Electrical Stimulation 
Device in Patients with Chronic Venous Disease. Eur J Vasc Endovasc Surg. 2017;53(1):114-121. doi:10.1016/j.ejvs.2016.09.015

29. Lai Q, Pretagostini R, Poli L, et al. Early Urine Output Predicts Graft Survival After Kidney Transplantation. Transplant Proc. 2010;42(4):1090-1092. doi:10.1016/j.transproceed.2010.03.088

30. Aquil S, Sharma H, Alharbi B, Pacoli K, Luke P, Sener A. The Impact of A Muscle Pump Activator on Incisional Wound Healing Compared to Standard Stockings and Compression Devices in Kidney and Kidney-pancreas Transplant Recipients: A Randomized Controlled Trial. Can Urol Assoc J. 2019;3.

31. Gero D, Gié O, Hübner M, Demartines N, Hahnloser D. Postoperative ileus: in search of an international consensus on definition, diagnosis, and treatment. Langenbeck's Arch Surg. 2017;402(1):149-158. doi:10.1007/s00423-016-1485-1

32. Sahin E, Terzioglu F. The Effect of Gum Chewing, Early Oral Hydration, and Early Mobilization on Intestinal Motility After Cesarean Birth. Worldviews Evidence-Based Nurs. 2015;12(6):380-388. doi:10.1111/wvn.12125

33. Raue W, Haase O, Junghans T, Scharfenberg M, Müller JM, Schwenk W. "Fast-track" multimodal rehabilitation program improves outcome after laparoscopic sigmoidectomy: A controlled prospective evaluation. Surg Endosc Other Interv Tech. 2004;18(10):14631468. doi:10.1007/s00464-003-9238-y

34. Partsch H, Blättler W. Compression and walking versus bed rest in the treatment of proximal deep venous thrombosis with low molecular weight heparin. J Vasc Surg. 2000;32(5):861-869. doi:10.1067/mva.2000.110352

35. Talec P, Gaujoux S, Samama CM. Early ambulation and prevention of post-operative thrombo-embolic risk. J Visc Surg. 2016;153(6):S11-S14. doi:10.1016/j.jviscsurg.2016.09.002

36. Alharbi B. The Effect of the GekoTM Device on Post Kidney and Pancreatic Transplantation Leg Edema. Electron Thesis Diss Repos. 2016;(January):4371.

37. Klaschik S, Gehlen J, Neumann C, et al. Network of Mediators for Vascular Inflammation and Leakage Is Dysbalanced during Cytoreductive Surgery for Late-Stage Ovarian Cancer. Mediators Inflamm. 2019;2019:1-9. doi:10.1155/2019/5263717 


\section{Figures and Tables}

Fig. 1. The The Geko ${ }^{\mathrm{TM}}$ (Firstkind Ltd, U.K.) device worn as a band over the legs bilaterally, just inferior to the fibular heads.

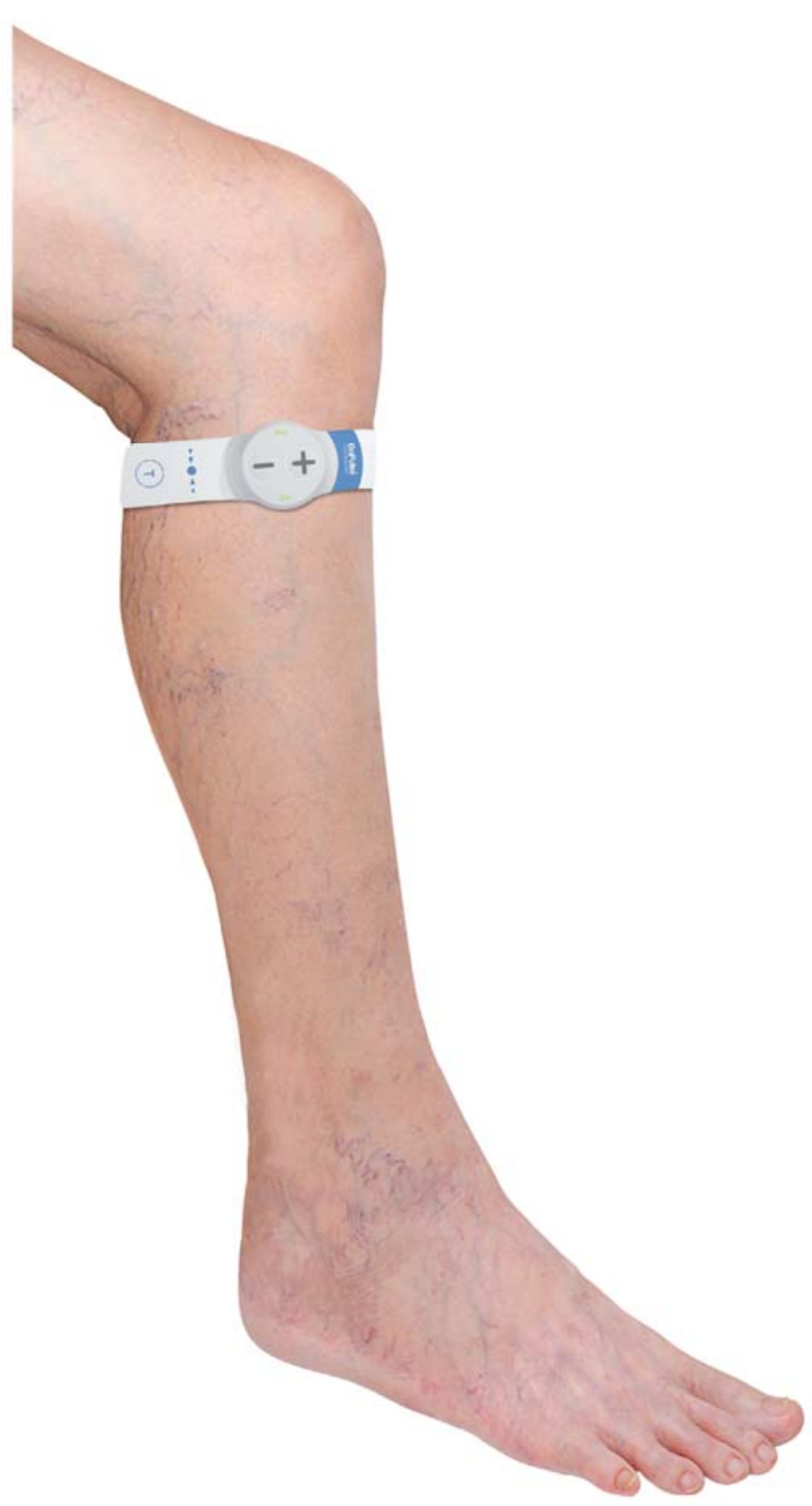


Fig. 2. Mean change in calf circumference between postoperative day 1 and 6. The MPA group had a significantly lower increase in calf circumference compared to the TED+IPC group $(2.5 \pm 1.5 \mathrm{~cm}$ vs $3.6 \pm 1.5 \mathrm{~cm} ; \mathrm{p}=0.001)$. IPC: intermittent pneumatic compression; MPA: muscle pump activator; TED: thrombo-embolic-deterrent.

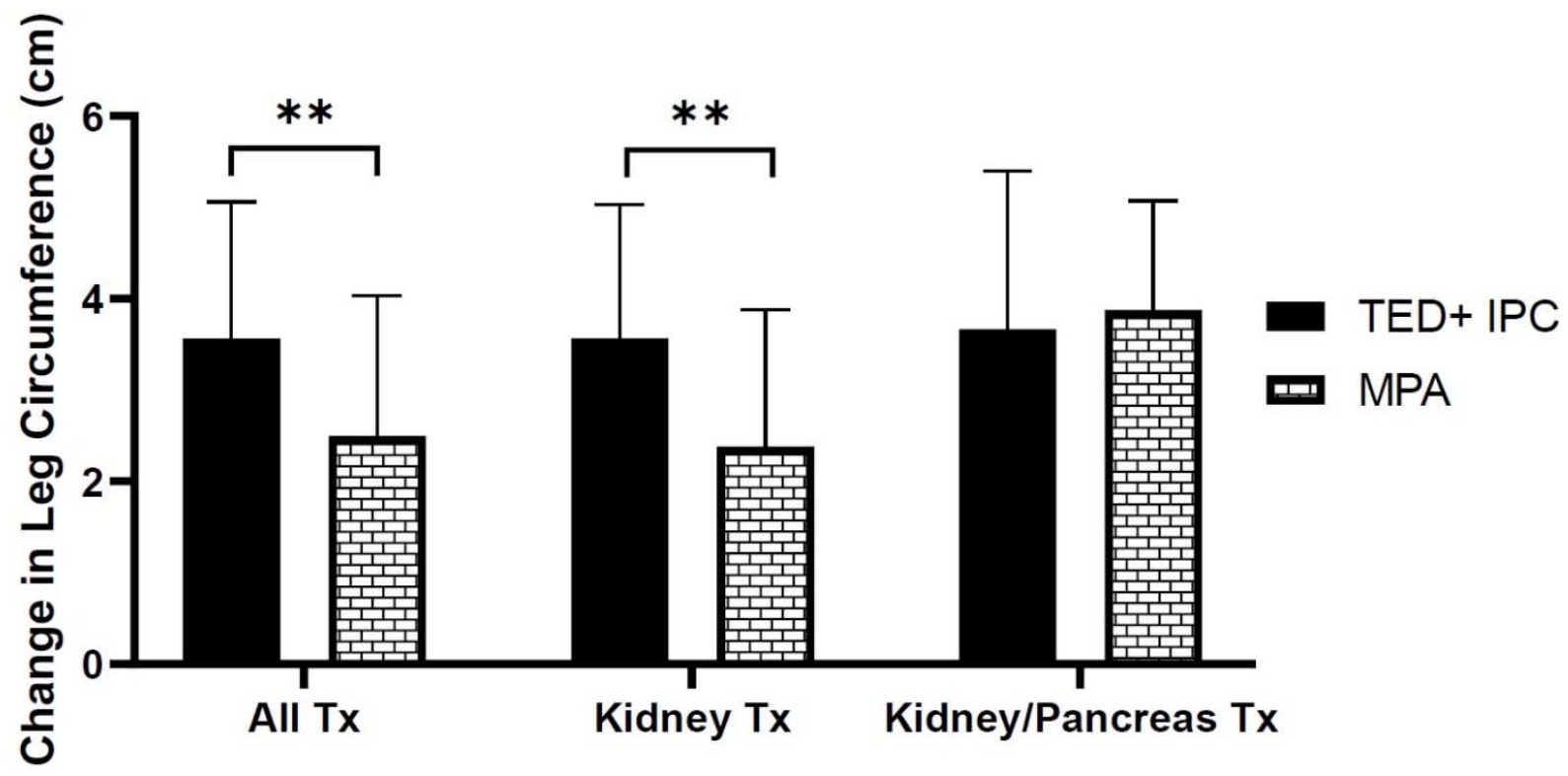

Fig. 3. Mean change in patient weight between post-operative day 1 and 6 . The MPA group had a significantly lower increase in calf circumference compared to the TED+IPC group (4.06 \pm 2.3 $\mathrm{kg}$ vs. $5.18 \pm 2.8 \mathrm{~kg}$; $\mathrm{p}=0.003$ ). IPC: intermittent pneumatic compression; MPA: muscle pump activator; TED: thrombo-embolic-deterrent.

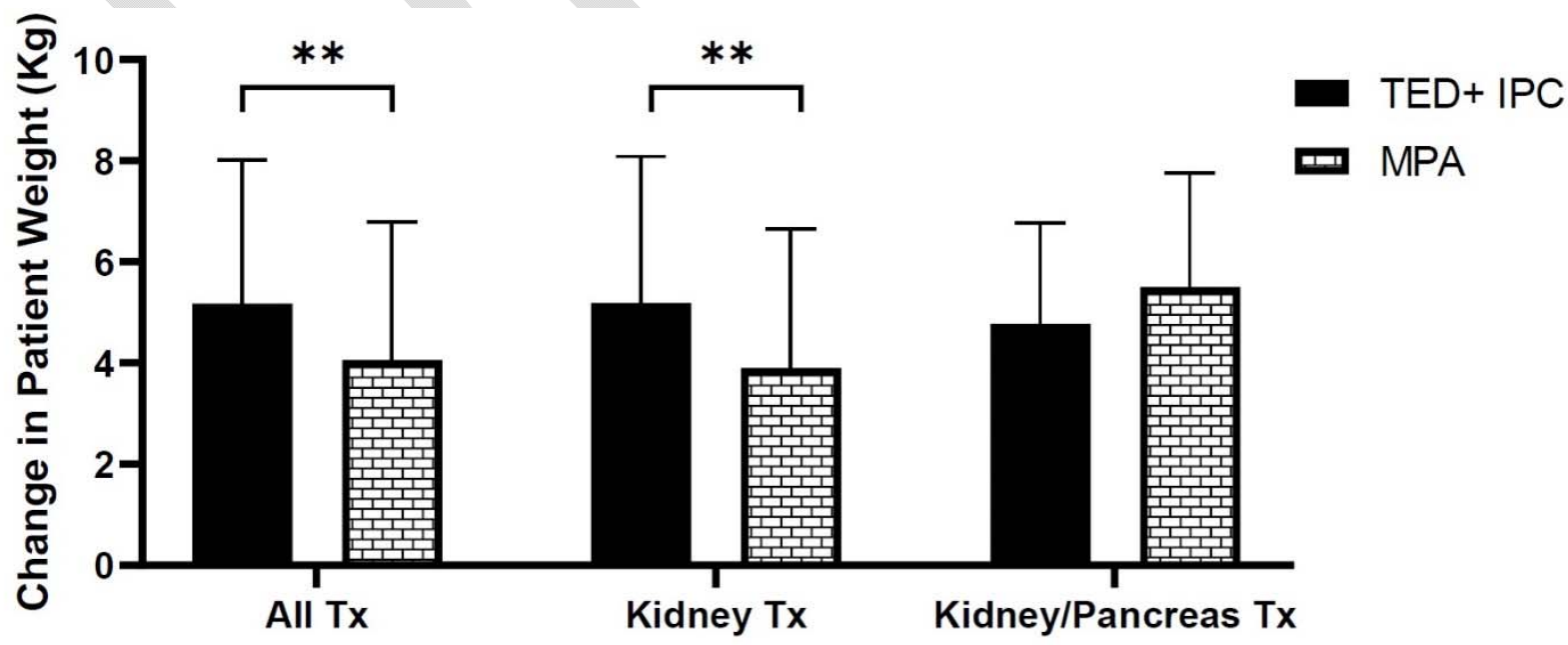


Fig. 4. Mean length of hospitalization. Participants in the MPA group had a significantly shorter hospital stay compared to the TED+IPC group (9.36 \pm 5.0 days vs $8.15 \pm 3.5$ days; $p=0.038$ ). No difference in length of hospitalization between the study groups was seen in SPK patients. IPC: intermittent pneumatic compression; MPA: muscle pump activator; TED: thrombo-embolicdeterrent.

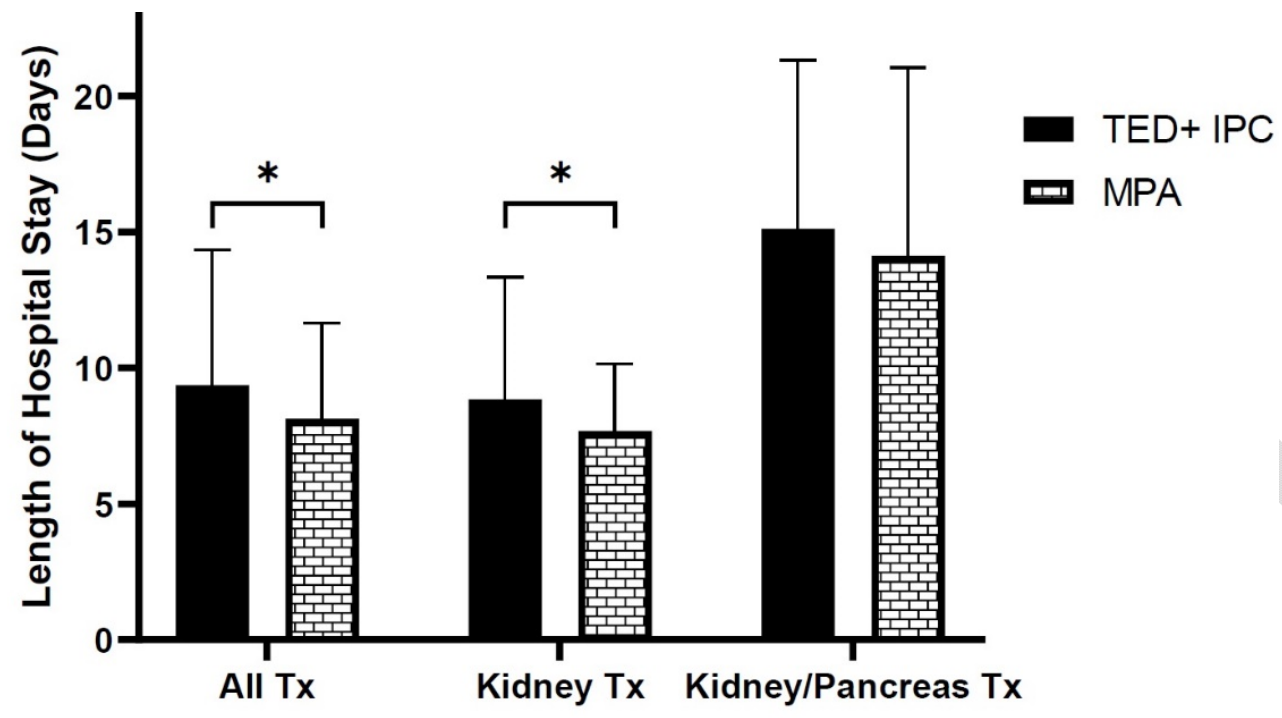

Fig. 5. Average number of steps taken on post-operative day 1 to 6 . The MPA group taken a significantly higher number of steps compared to the TED+IPC group (1231 189 steps $4.06 \pm 2.3 \mathrm{~kg}$ vs $1099 \pm 249$ steps, $\mathrm{p}=0.009)$. IPC: intermittent pneumatic compression; MPA: muscle pump activator; TED: thrombo-embolic-deterrent.

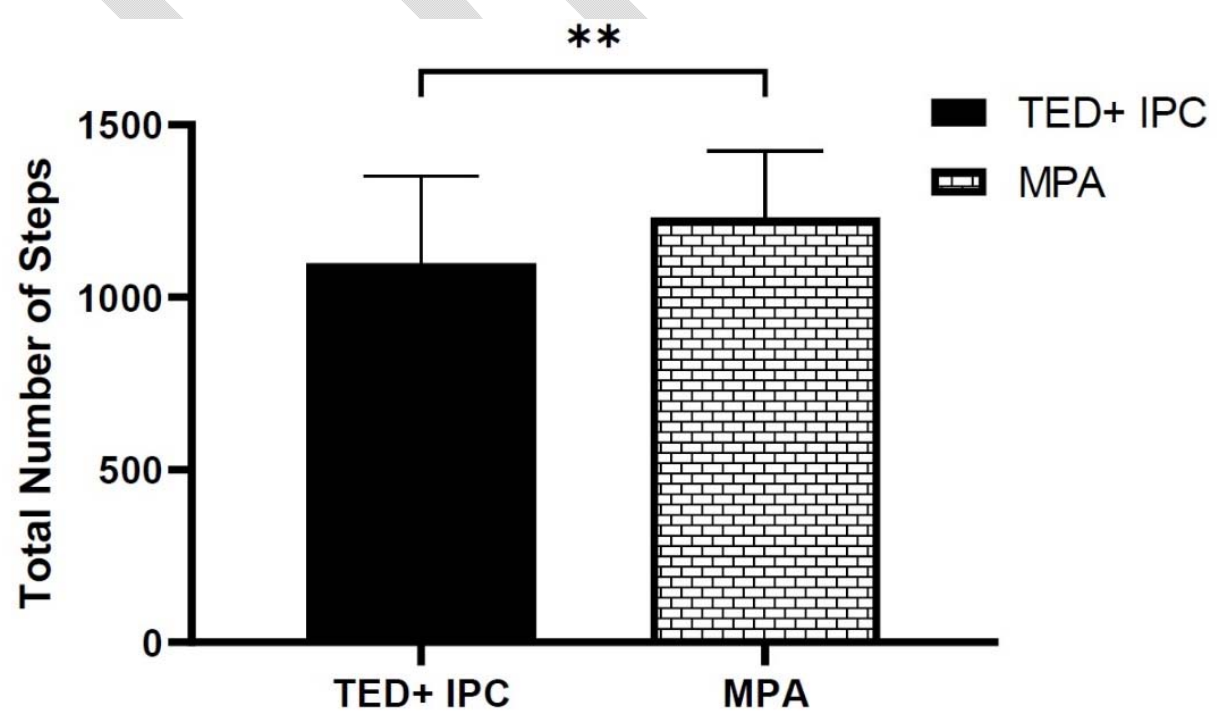




\begin{tabular}{|c|c|c|c|}
\hline & \multicolumn{2}{|c|}{ Recipient intervention } & \multirow[b]{2}{*}{$\mathbf{p}$} \\
\hline & TED+IPC & MPA & \\
\hline Number of participants & 111 & 110 & - \\
\hline Age, years & $51.5 \pm 13.4$ & $52.9 \pm 13.2$ & 0.43 \\
\hline Male:female & $64: 47$ & $69: 41$ & 0.44 \\
\hline Weight & $77.47 \pm 16.9$ & $78.71 \pm 17.6$ & 0.59 \\
\hline BMI $\left(\mathrm{kg} / \mathrm{m}^{2}\right)$ & $27.39 \pm 5.2$ & $27.02 \pm 4.7$ & 0.58 \\
\hline Type of dialysis & & & 0.79 \\
\hline HD & 69 & 67 & \\
\hline PD & 30 & 28 & 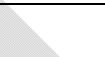 \\
\hline Pre-emptive & 12 & 15 & 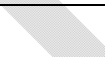 \\
\hline Type of donor & & 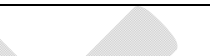 & 0.27 \\
\hline LD & 30 & 22 & \\
\hline DBD & 55 & 54 & \\
\hline DCD & 26 & 34 & \\
\hline Cause of ESRD & & & 0.98 \\
\hline $\mathrm{DN}$ & 37 & 26 & \\
\hline HTNN & 12 & 9 & \\
\hline $\mathrm{DN}+\mathrm{HTNN}$ & 0 & 8 & \\
\hline IgA nephropathy & 8 & 10 & \\
\hline Alport syndrome & 2 & 2 & \\
\hline Anatomic & 7 & 5 & \\
\hline FSGS & 4 & 5 & \\
\hline PCKD & 10 & 15 & \\
\hline Other autoimmune & 10 & 8 & \\
\hline Drug-induced & 3 & 5 & \\
\hline GN/NS & 6 & 7 & \\
\hline Congenital/genetic & 5 & 4 & \\
\hline Distributive/systemic shock & 4 & 1 & \\
\hline Unknown/other & 3 & 5 & \\
\hline
\end{tabular}

Anatomic includes urinary tract obstruction and reflux. DBD: donor after brain death; DCD: donor after cardiac death; DN: diabetic nephropathy; FSGS: focal segmental glomerulosclerosis; GN/NS: glomerulonephritis/nephrotic syndrome; HTNN: hypertensive nephrosclerosis; LD: living donor; PCKD: polycystic kidney disease. 


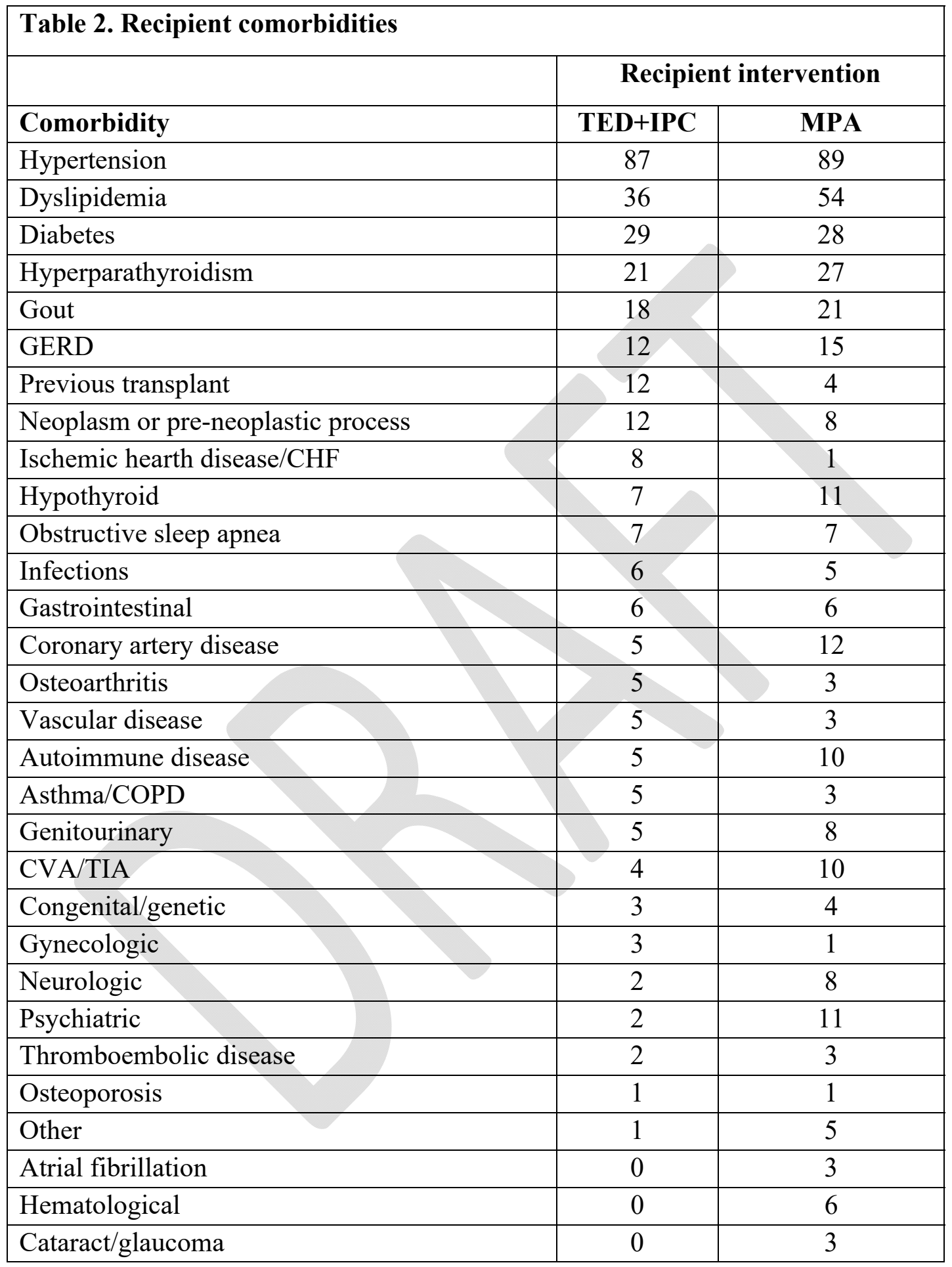

CHF: congestive heart failure; COPD: chronic obstructive pulmonary disease; CVA: cerebral vascular accident; GERD: gastroesophageal reflux disease; IPC: intermittent pneumatic compression; MPA: muscle pump activator; TED: thrombo-embolic-deterrent; TIA: transient ischemic attack. 


\begin{tabular}{|l|c|c|c|}
\hline Table 3. Urine output between postoperative day 1-6 \\
\hline & \multicolumn{2}{|c|}{ Urine output (mL) } & \\
\hline & TED+IPC & MPA & p \\
\hline All Tx & 12595 & 15986 & 0.004 \\
\hline Kidney Tx & 12457 & 16325 & 0.015 \\
\hline Kidney/pancreas Tx & 4933 & 9662 & 0.98 \\
\hline
\end{tabular}

The MPA group had significantly higher urine output in kidney transplant recipients but not SPK recipients. IPC: intermittent pneumatic compression; MPA: muscle pump activator; SPK: simultaneous pancreas-kidney; TED: thrombo-embolic-deterrent; Tx: treatment.

\begin{tabular}{|l|c|c|c|}
\hline \multicolumn{4}{|l|}{ Table 4. Primary endpoints analysis by donor type } \\
\hline & TED+IPC & MPA & p \\
\hline Living donor & & & \\
\hline Urine output $(\mathrm{mL})$ & 18902 & 23989 & 0.009 \\
\hline Weight $(\mathrm{Kg})$ & 4.90 & 2.82 & 0.005 \\
\hline Calf size $(\mathrm{cm})$ & 3.23 & 2.27 & 0.010 \\
\hline DBD & & & \\
\hline Urine output $(\mathrm{mL})$ & 12832.13 & 19292.33 & $<0.001$ \\
\hline Weight $(\mathrm{Kg})$ & 4.87 & 3.91 & 0.05 \\
\hline Calf size $(\mathrm{cm})$ & 3.65 & 2.26 & $<0.001$ \\
\hline DCD & & & \\
\hline Urine output $(\mathrm{mL})$ & 4815.50 & 5496.38 & 0.27 \\
\hline Weight $(\mathrm{Kg})$ & 6.04 & 4.97 & 0.23 \\
\hline Calf size $(\mathrm{cm})$ & 3.81 & 3.00 & 0.08 \\
\hline
\end{tabular}

Urine output, weight and calf size were significantly different between the TED+IPC and the MPA recipients for those who received living donor or DBD donor kidneys. There was no difference in these measures seen between study groups for recipients who received DCD donor kidneys. DBD: donor after brain death; DCD: donor after cardiac death; IPC: intermittent pneumatic compression; MPA: muscle pump activator; TED: thrombo-embolic-deterrent. 